EXPERIMENTAL STUDY

\title{
Effect of insulin on nitric oxide synthase-like immunostaining of arteries in various organs in Zucker diabetic fatty rats
}

\author{
Mikiko Kawaguchi, Kunio Koshimura, Motoi Sohmiya, Yoshio Murakami, Tatsuo Gonda ${ }^{1}$ and Yuzuru Kato \\ First Division, Department of Medicine and ${ }^{1}$ Institute of Experimental Animals, Shimane Medical University, Izumo, Japan \\ (Correspondence should be addressed to M Kawaguchi, First Division, Department of Medicine, Shimane Medical University, 89-1 Enya-cho, \\ Izumo 693-8501, Japan; Email: k-mikiko@shimane-med.ac.jp)
}

\begin{abstract}
Objective: Recently we reported that insulin treatment improved hypertension by inducing nitric oxide synthase (NOS) in Zucker diabetic fatty (ZDF) rats. In the present study, we investigated subtypes of NOS induced by insulin in arteries in various organs of ZDF rats using immunohistochemistry.

Design: Following treatment with insulin, localization of two subtypes of NOS in the arterial tissues of various organs was identified.

Methods: Following 4 weeks of s.c. injection of insulin, the aorta, cerebral cortex, pancreas and kidney were stained with polyclonal anti-endothelial NOS (eNOS) or anti-inducible NOS (iNOS) antibodies. Results: In the aortic tissue, eNOS-like immunostaining was observed equally in the insulin-treated group and the control group, whereas iNOS-like immunostaining was present more densely in the insulin-treated group. In the cerebral artery, eNOS-like immunostaining was observed in the endothelium and was enhanced in the insulin-treated group. In the control group, iNOS-like immunostaining was absent in the cerebral artery, whereas immunostaining was densely observed in the insulin-treated group. In the interlobular artery of the pancreas, both eNOS-like and iNOS-like immunostaining was present in the control group and was enhanced in the insulin-treated group. In kidney, both eNOS-like and iNOS-like immunostaining was more densely present in the arterial tissue of the insulin-treated group.

Conclusions: These results taken together suggest that insulin treatment induced NOS in arteries in various organs and that iNOS was more strongly induced than eNOS by insulin treatment in the ZDF rat.

European Journal of Endocrinology 145 343-349
\end{abstract}

\section{Introduction}

Recently we have reported that insulin treatment improved hypertension in Zucker diabetic fatty (ZDF) rats $(1,2)$, a model of type 2 diabetes mellitus (3). The antihypertensive effect of insulin was explained either by the secondary action of improved glucose metabolism after insulin treatment or by the direct action on peripheral arterial vessels (4-11). Several lines of evidence suggest that insulin stimulates nitric oxide (NO) generation in endothelial cells (12-15). We observed that serum levels of NO metabolites were increased by insulin treatment in the ZDF rat $(1,2)$. Furthermore, NO synthase (NOS) activity and NADPH diaphorase staining were enhanced by insulin treatment (2). These data suggest that insulin treatment induces NOS in the ZDF rat. However, NADPH diaphorase staining does not always coincide with the presence of NOS (16). In the present study, we further investigated the effect of insulin on induction of NOS in arteries of various organs in the ZDF rat using polyclonal antibodies against endothelial NOS (eNOS) and inducible NOS (iNOS).

\section{Materials and methods}

\section{Antibodies and chemicals}

Rabbit polyclonal anti-bovine eNOS antibody (antieNOS ab; B14553) was purchased from CalbiochemNovabiochem (San Diego, CA, USA). Rabbit polyclonal anti-mouse iNOS antibody (anti-iNOS ab; PA3-030) was purchased from Affinity BioReagents (Golden, CO, USA). Both antibodies showed no cross-reactivity with other subtypes of NOS. All other chemicals were of the purest grade available from regular commercial sources.

\section{Animals and insulin treatment}

Eight male ZDF rats were obtained at the age of 7 weeks from Genetic Models Inc. (Indianapolis, IN, USA), 
Table 1 Summary of the effect of insulin on immunostaining of eNOS and iNOS in various tissues on ZDF rats.

\begin{tabular}{lccc}
\hline Tissues & NOS isozymes & Control & Insulin \\
\hline Aorta & & & \\
Endothelium & eNOS & ++ & ++ \\
Smooth muscle & iNOS & ++ & ++ \\
& eNOS & \pm & \pm \\
Cerebral artery & iNOS & \pm & ++ \\
Endothelium & eNOS & \pm & ++ \\
Smooth muscle & iNOS & - & + \\
eNOS & - & - \\
Pancreas & iNOS & - & ++ \\
Interlobular artery & eNOS & \pm & ++ \\
& iNOS & + & ++ \\
Exocrine cells & eNOS & - & - \\
Kidney & iNOS & - & \pm \\
Arterial endothelium & eNOS & \pm & ++ \\
Arterial smooth muscle & iNOS & - & \pm \\
Collecting tubule & iNOS & - & + \\
& eNOS & + & ++ \\
& iNOS & ++ & ++ \\
& & + & + \\
\hline
\end{tabular}

- , not stained; \pm , slightly stained; + , moderately stained; ++ , densely stained.
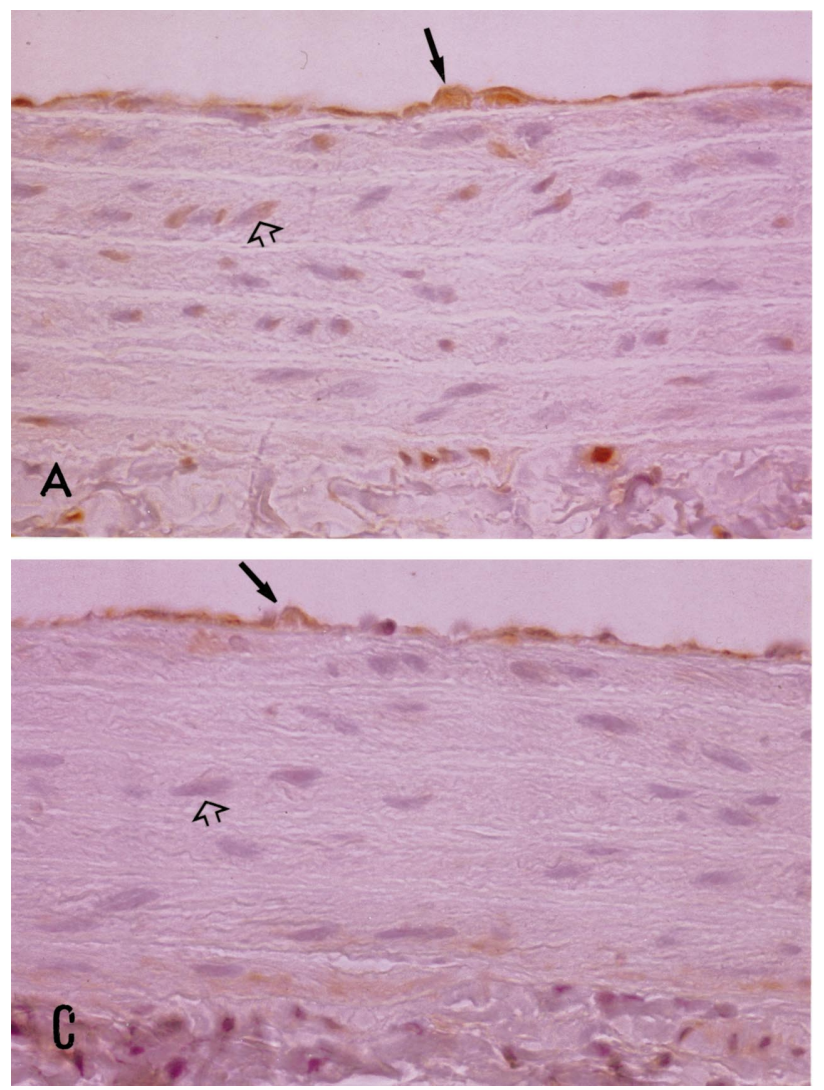

housed in pairs, and kept in artificial lighting (lights on $0800 \mathrm{~h}$, off $2000 \mathrm{~h}$ ). Food and tap water were freely given until the age of 12 weeks. The rats were randomly divided into two groups, the insulin-treated group and the control group. Insulin therapy was started at the age of 12 weeks when plasma glucose levels were over $200 \mathrm{mg} / \mathrm{ml}$. Insulin was injected s.c. in a dose of $25 \mathrm{U} / \mathrm{kg}$ per day between 1700 and $1800 \mathrm{~h}$ at $24 \mathrm{~h}$ intervals during the subsequent 4 weeks as previously described (17). In the control group, saline solution was injected s.c. in a dose of $1.25 \mathrm{ml} / \mathrm{kg}$ per day. During the experimental period, food and water intake as well as body weight were monitored at 1800 h every day.

\section{Anti-NOS immunostaining}

After treatment for 4 weeks, rats were anesthetized i.p. with sodium pentobarbital $(50 \mathrm{mg} / \mathrm{kg})$ and perfused with $300 \mathrm{ml} 0.9 \%$ saline followed by $600 \mathrm{ml} 4 \%$ paraformaldehyde in $0.1 \mathrm{M}$ phosphate buffer ( $\mathrm{pH}$ 7.4) via the left ventricle. The whole aorta, defined by the aortic arch through the bifurcation of the iliac artery, whole brain and kidney
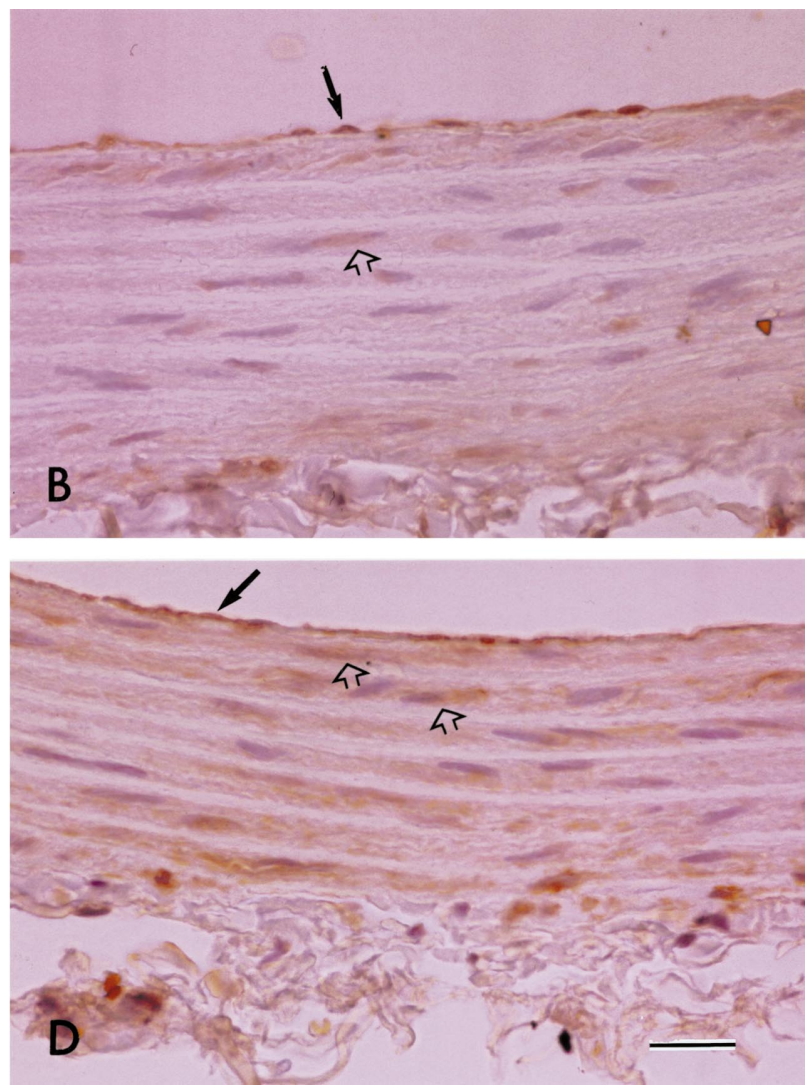

Figure 1 Photomicrographs of NOS-like immunostaining of the aorta from ZDF rats. (A) eNOS-like immunostaining in control rats. (B) eNOS-like immunostaining in insulin-treated rats. (C) iNOS-like immunostaining in control rats. (D) iNOS-like immunostaining in insulin-treated rats. Solid arrows indicate endothelial cells, open arrows smooth muscle cells. Scale bar represents $20 \mu \mathrm{m}$. Representative sections are shown. Similar results were obtained from all rats examined. 
were removed and immersed in the same fixative at $4{ }^{\circ} \mathrm{C}$ overnight. The pancreases was removed from each decapitated rat without perfusion and immersed in the same fixative as the other organs at $4{ }^{\circ} \mathrm{C}$ overnight. The fixed tissues were washed with water for $3 \mathrm{~h}$ and dehydrated in graded ethanol $(70,90,99$ and $100 \%$ ) and chloroform, and embedded in 10\% paraformaldehyde. The paraffin-embedded tissues were cut into $5 \mu \mathrm{m}$ thick serial sections and placed on polyL-lysine-coated glass slides. The paraffin-embedded serial sections were deparaffinized in 100\% xylene, then rehydrated in graded ethanol with a step-wise decrease in concentration $(99,95,90,80$ and $70 \%)$ and water. After microwave treatment in $10 \mathrm{mM}$ citrate buffer for $5 \mathrm{~min}$ twice, the sections were incubated in $0.3 \% \mathrm{H}_{2} \mathrm{O}_{2}$ in $100 \%$ methanol for $10 \mathrm{~min}$ at room temperature to block endogenous peroxidase. The sections were washed in PBS and nonspecific binding was blocked by $30 \mathrm{~min}$ incubation in $1 \%$ normal goat serum in PBS at room temperature, and then the sections were incubated with anti-eNOS ab or anti-iNOS ab at $4{ }^{\circ} \mathrm{C}$ overnight. Anti-eNOS ab and anti-iNOS ab were diluted in PBS at 1:1000 and 1:750
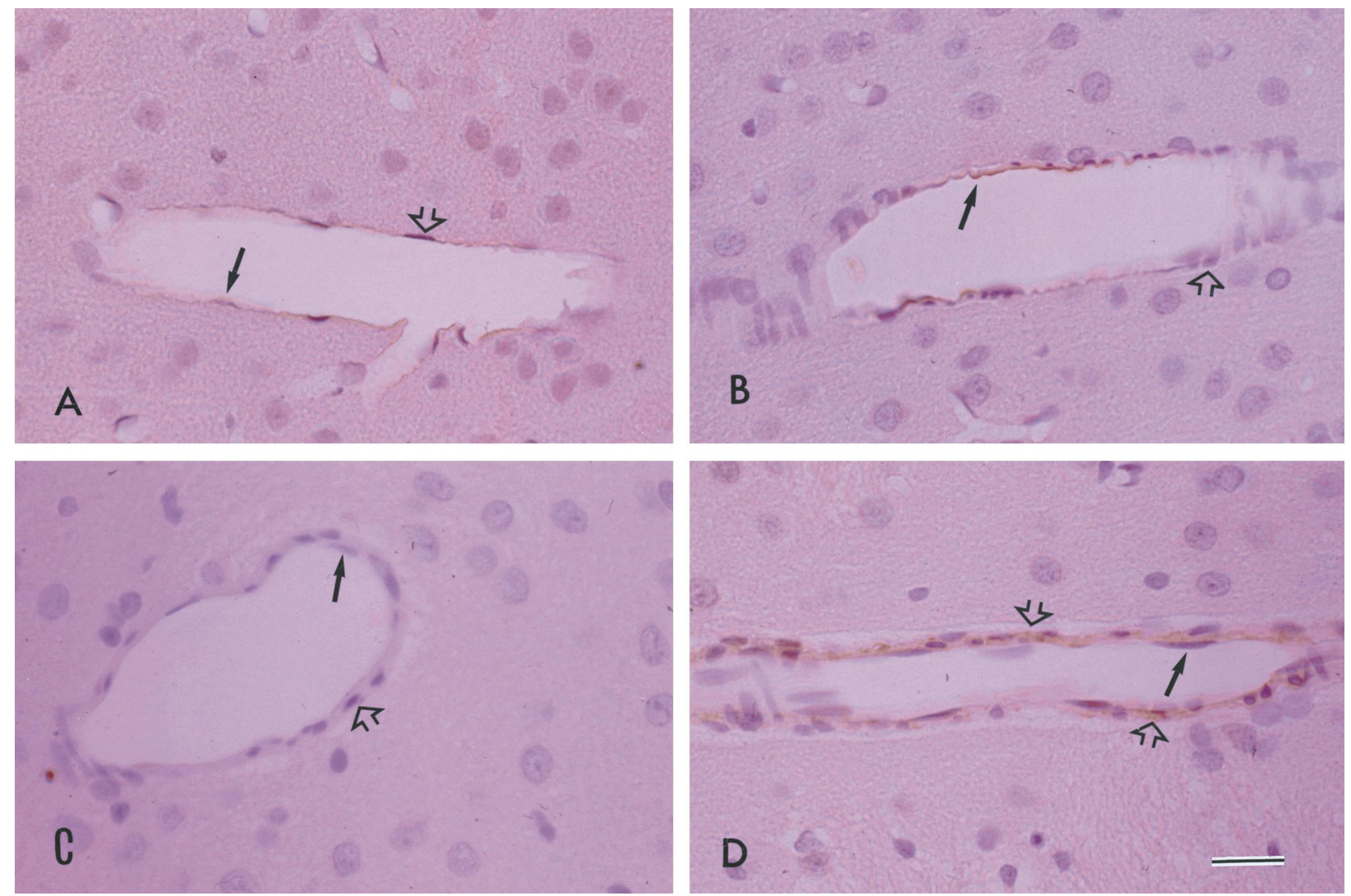

Figure 2 Photomicrographs of NOS-like immunostaining of the cerebral artery from ZDF rats. (A) eNOS-like immunostaining in control rats. (B) eNOS-like immunostaining in insulin-treated rats. (C) iNOS-like immunostaining in control rats. (D) iNOS-like immunostaining in insulin-treated rats. Solid arrows indicate endothelial cells, open arrows smooth muscle cells. Scale bar represents $20 \mu \mathrm{m}$. Representative sections are shown. Similar results were obtained from all rats examined. 


\section{Results}

To investigate the mechanism of antihypertensive effect of insulin, we studied immunostaining of NOS exclusively in arteries of examined organs. Since blank staining for eNOS and iNOS staining was negligible, positive cells for eNOS-like and iNOS-like immunostaining were definitely identified in all the sections of ZDF rats. The results of NOS-like immunostaining in the arteries of the examined organs are summarized in Table 1. In the ZDF rat, insulin treatment enhanced staining of NOS, especially iNOS, in the arteries of all the examined organs.

\section{Aorta}

In the control group, the endothelium was densely stained by anti-eNOS ab and the smooth muscle was slightly stained (Fig. 1A). The eNOS-like immunostaining was not changed in the insulin-treated group (Fig. 1B). The iNOS-like immunostaining was densely detected in the endothelium and slightly in the smooth
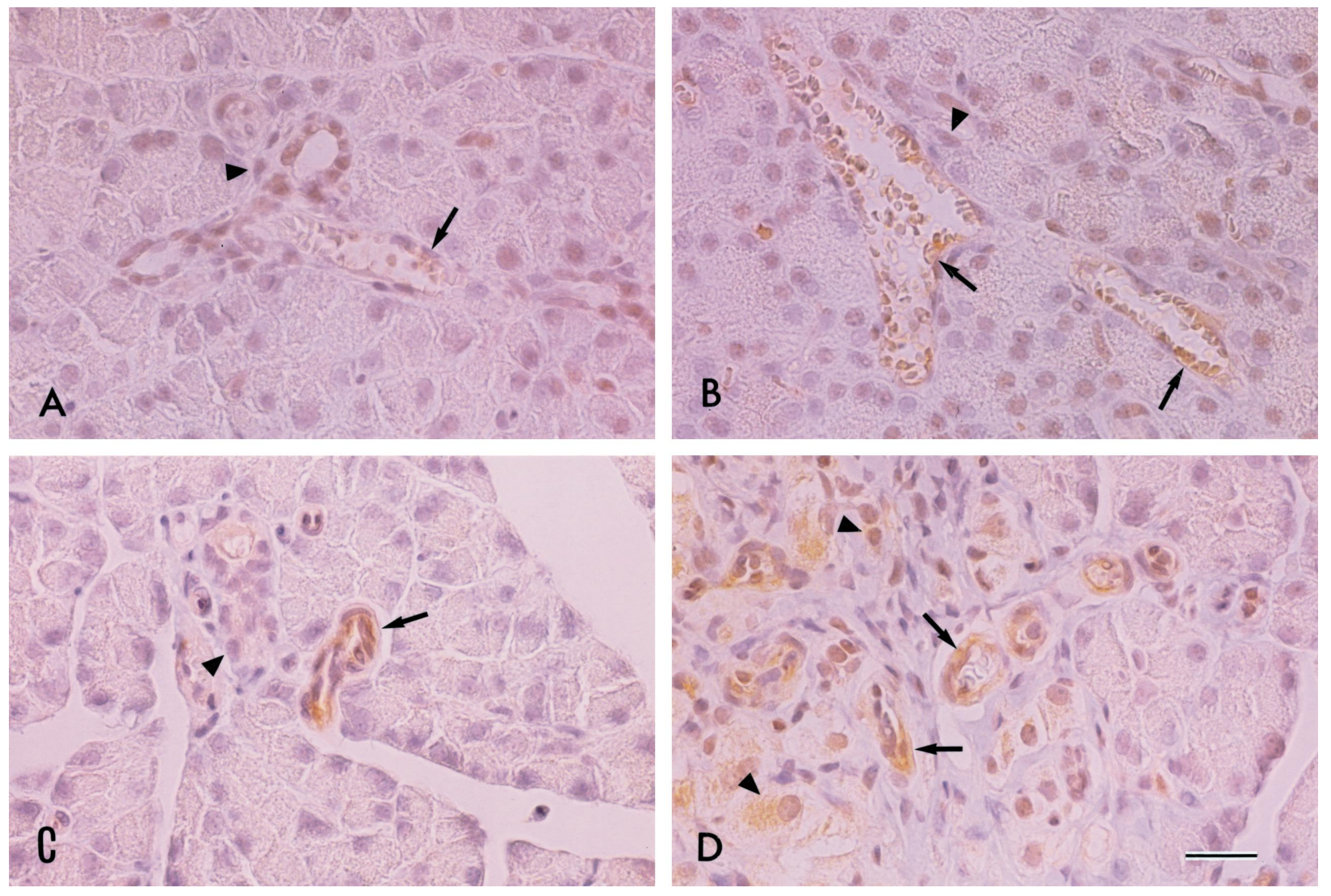

Figure 3 Photomicrographs of NOS-like immunostaining of the pancreas from ZDF rats. (A) eNOS-like immunostaining of interlobular artery from control rats. (B) eNOS-like immunostaining of interlobular artery from insulin-treated rats. (C) iNOS-like immunostaining of interlobular artery from control rats. (D) iNOS-like immunostaining of interlobular artery and endocrine cells from insulin-treated rats. Arrows indicate arterial cells, arrow-heads endocrine cells. Scale bar represents $20 \mu \mathrm{m}$. Representative sections are shown. Similar results were obtained from all rats examined. 
the insulin-treated group, eNOS-like immunostaining of the interlobular artery was enhanced (Fig. 3B). Dense iNOS-like immunostaining was observed in the interlobular artery of both groups (Fig. 3C and D). In both groups, eNOS-like immunostaining was not detected in the endocrine cells (Fig. 3A and B). In the control group, iNOS-like immunostaining of the endocrine cells was absent (Fig. 3C), whereas it was densely detected in the insulin-treated group (Fig. 3D).

\section{Kidney}

In the control group, eNOS-like immunostaining was slightly detected in the arterial endothelium but not in the smooth muscle (Fig. 4A). Treatment with insulin enhanced eNOS-like immunostaining of both the arterial endothelium and the smooth muscle (Fig. 4B). In the control group, iNOS-like immunostaining was absent in the endothelium but moderately detected in the smooth muscle (Fig. 4C). In the insulin-treated group, iNOS-like immunostaining was slightly detected in the endothelium and densely in the smooth muscle
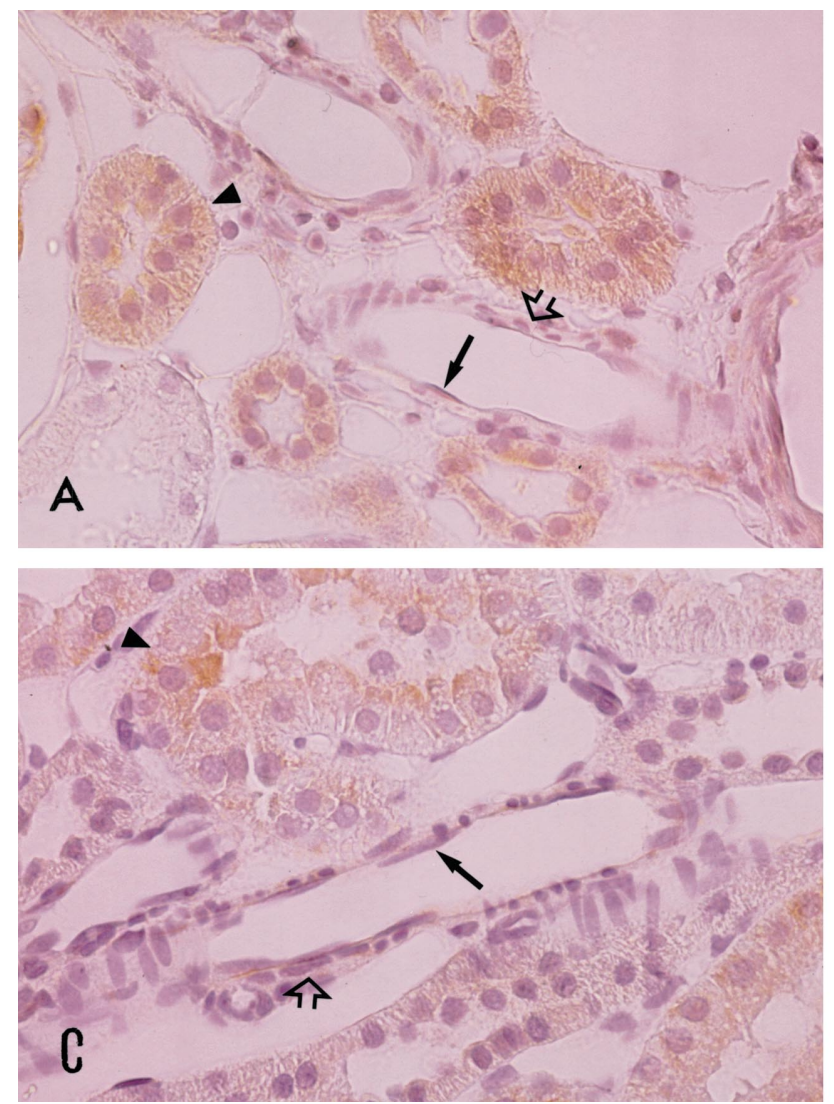

(Fig. 4D). The collecting tubule was densely positive for eNOS-like immunostaining both in the control group (Fig. 4A) and in the insulin-treated group (Fig. 4B). In the collecting tubules, iNOS-like immunostaining was moderately detected in both groups.

\section{Discussion}

In the control group, eNOS-like immunostaining was identified only in endothelium of the arteries, whereas iNOS-like immunostaining was observed in both endothelium and smooth muscle of arteries, as previously reported $(16,18-20)$. The present study demonstrates that insulin treatment enhanced eNOSlike immunostaining in endothelium and iNOS-like immunostaining in endothelium and smooth muscle of arteries in the various organs in the ZDF rat. These results were consistent with previous finding of a study with cultured human endothelial cells and smooth muscle cells (21). However, Kuboki et al. (22) reported that insulin treatment did not induce eNOS mRNA in microvessels taken from ZDF rats. The discrepancy may be due to difference in experimental conditions. In
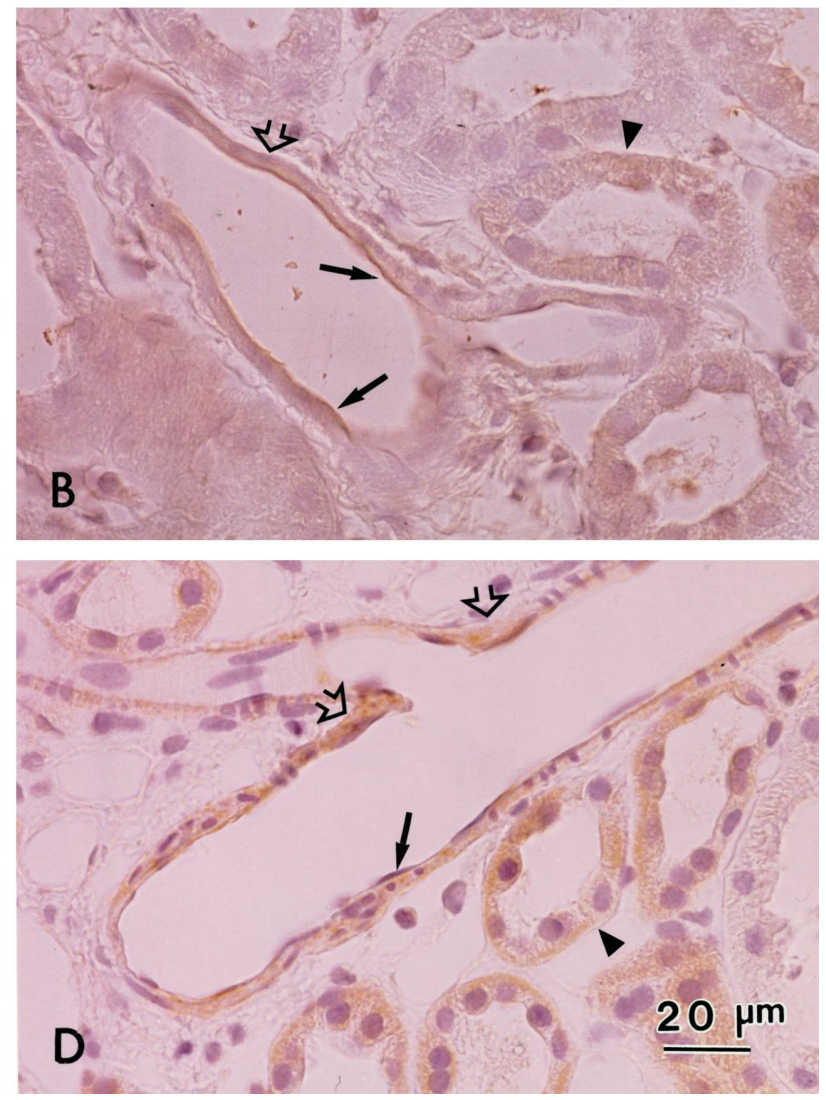

Figure 4 Photomicrographs of NOS-like immunostaining of the kidney from ZDF rats. (A) eNOS-like immunostaining of artery and collecting tubules from control rats. (B) eNOS-like immunostaining of artery and collecting tubules from insulin-treated rats. (C) iNOSlike immunostaining of artery from control rats. (D) iNOS-like immunostaining of artery from insulin-treated rats. Solid arrows indicate endothelial cells, open arrows smooth muscle cells. Arrow-heads indicate collecting tubules. Representative sections are shown. Similar results were obtained from all rats examined. 
contrast to our in vivo experiment, they studied the effect of insulin under in vitro conditions. Moreover, in the present study, insulin was administered for 4 weeks, whereas in their study, microvessels were incubated with insulin for $6 \mathrm{~h}$. In the kidney, eNOS was densely stained in arterial smooth muscle of the insulin-treated group. This exceptional finding in the present study remains to be explained.

Insulin-induced NOS-like immunostaining in the arteries supported our previous observation that insulin-treatment induced NOS in aorta of ZDF rats as studied by NADPH diaphorase staining (2). The present data indicate that improvement of hypertension by insulin treatment was due to induction of NOS in the arteries in ZDF rats. In ZDF rats, insulin-induced NOS-like immunostaining was predominantly iNOS-like immunostaining. It is possible that insulin treatment stimulated NO production through iNOS induction in ZDF rats. Incubation of microvessels of non-diabetic rats with insulin for $6 \mathrm{~h}$ induced eNOS mRNA (22). However, it remains to be clarified whether chronic insulin treatment induces NOS under non-diabetic conditions. The present study suggests that insulin treatment improved general blood flow by inducing NOS in the arteries in the ZDF rat. It is speculated that NOS induction in the renal arteries improves renal blood flow and dysfunction of the renin-angiotensin system under hypertensive condition $(23,24)$. Although we did not examine the renin-angiotensin system in the ZDF rat, it is possible that insulin improves hypertension partly by reducing resistance of renal arterioles and serum renin level in these rats. The present data suggest that insulin treatment improves cerebral blood flow in the ZDF rat. It is assumed that central control of blood pressure was modulated by insulin treatment in the ZDF rat. In addition to arteries, insulin treatment induced NOS in pancreatic exocrine cells and renal tubules. The effects of insulin-induced NOS on pancreatic functions and renal functions remain to be investigated.

The present study suggests that in the ZDF rat insulin treatment affects various physiological conditions including glucose metabolism and blood pressure partly by induction of NOS.

\section{Acknowledgements}

This work was supported in part by grants from the Ministry of Education and Culture, Japan, the Ministry of Health and Welfare, Japan, and the Shimane Institute for Intractable Diseases. We thank Mrs A Kanayama for her technical assistance and Mrs A Kawakami for her secretarial help.

\section{References}

1 Kawaguchi M, Koshimura K, Murakami Y \& Kato Y. Antihypertensive effect of insulin in the Zucker diabetic fatty (ZDF) rat, an animal model for non-insulin dependent diabetes mellitus with insulin resistance. Biomedical Research 199920 57-59.

2 Kawaguchi M, Koshimura K, Murakami Y, Tsumori M, Gonda T \& Kato Y. Antihypertensive effect of insulin via nitric oxide production in the Zucker diabetic fatty (ZDF) rat, an animal model for non-insulin-dependent diabetes mellitus. European Journal of Endocrinology 1999140 341-349.

3 Clark JB, Palmer CJ \& Shaw WN. The diabetic Zucker fatty rat. Proceedings of the Society for Experimental Biology and Medicine $198317368-75$.

4 Hall JE, Brands MW, Kivlighn SD, Mizelle HL, Hildebrandt DA \& Gaillard CA. Chronic hyperinsulinemia and blood pressure: interaction with catecholamine? Hypertension 199015 519-527.

5 Anderson EA, Hoffman RP, Balon TW, Sinkey CA \& Mark AL. Hyperinsulinemia produces both sympathetic neural activation and vasodilatation in normal humans. Journal of Clinical Investigation $1991872246-2252$.

6 Anderson EA \& Mark AL. The vasodilator action of insulin: implications for the insulin hypothesis of hypertension. Hypertension 199321 136-141.

7 Baron AD. Hemodynamic actions of insulin. American Journal of Physiology 1994267 E187-E202.

8 Scott AR, Bennet T \& Macdonald IA. Effects of hyperinsulinemia on the cardiovascular responses to graded hypovolemia in normal and diabetic subjects. Clinical Science 198875 85-92.

9 Natali A, Buzzigoli G, Taddei S, Santori D, Cerri M, Pedrinelli R et al. Effects of insulin on hemodynamics and metabolism in human forearm. Diabetes 199039 490-500.

10 Anderson EA, Hoffmann RP, Balon TW, Sinkey CA \& Mark AL. Hyperinsulinemia in humans increases muscle sympathetic nerve activity, but reduces forearm vascular resistance. Clinical Research $199020219-225$.

11 Vollenweider P, Tappy L, Randin D, Schneiter P, Jequier E, Nicod P et al. Differential effects of hyperinsulinemia and carbohydrate metabolism on sympathetic nerve activity and muscle blood flow in humans. Journal of Clinical Investigation 199392 147-154.

12 Sobrevia L, Nadal A, Yudilevich DL \& Mann GE. Activation of l-arginine transport (system $\mathrm{y}+$ ) and nitric oxide synthase by elevated glucose and insulin in human endothelial cells. Journal of Physiology 1996490 775-781.

13 Zeng G \& Quon MJ. Insulin-stimulated production of nitric oxide is inhibited by wortmannin: direct measurement in vascular endothelial cells. Journal of Clinical Investigation $1996 \mathbf{9 8}$ 894-898.

14 Tsukahara H, Kikuchi K, Tsumura K, Kimura K, Hata I, Hiraoka $\mathrm{M}$ et al. Experimentally induced acute hyperinsulinemia stimulates endogenous nitric oxide production in humans: detection using urinary $\mathrm{NO}_{2}{ }^{-} / \mathrm{NO}_{3}{ }^{-}$excretion. Metabolism 199746 406-409.

15 Kawaguchi M, Koshimura K, Murakami Y, Tsumori M \& Kato Y. Effects of insulin on nitric oxide production in cultured human endothelial cells. Shimane Journal of Medical Sciences 199917 9-13.

16 Young HM, O'Brien AJ, Furness JB, Ciampoli D, Harwick JP, McCabe TJ et al. Relationships between NADPH diaphorase staining and neuronal, endothelial, and inducible nitric oxide synthase and cytochrome $\mathrm{P} 450$ reductase immunoreactivities in guinea-pig tissues. Histochemistry and Cell Biology $1997 \mathbf{1 0 7}$ $19-29$.

17 Tanigawa K, Kawaguchi M, Tanaka O \& Kato Y. Skeletal malformations in rat offspring: long-term effect of maternal insulin-induced hypoglycemia during organogenesis. Diabetes 199140 1115-1121.

18 Pollock JS, Nakane M, Buttery LD, Martinez A, Springall D, Polak JM et al. Characterization and localization of endothelial nitric oxide synthase using specific monoclonal antibodies. American Journal of Physiology 1993265 C1379-C1387.

19 Akyürek LM, Fellström BC, Yan Z-Q, Hansson GK, Funa K \& Larsson E. Inducible and endothelial nitric oxide synthase 
expression during development of transplant arteriosclerosis in rat aortic grafts. American Journal of Pathology 1996149 $1981-1990$.

20 Liu SF, Barnes PJ \& Evans TW. Time course and cellular localization of lipopolysaccharide-induced inducible nitric oxide synthase messenger RNA expression in the rat in vivo. Critical Care Medicine 199725 512-518.

21 Aljada A \& Dandona P. Effect of insulin on human aortic endothelial nitric oxide. Metabolism 200049 147-150.

22 Kuboki K, Jiang ZY, Takahara N, Ha SW, Igarashi M, Yamauchi T et al. Regulation of endothelial constitutive nitric oxide synthase gene expression in endothelial cells and in vivo: a specific vascular action of insulin. Circulation $2000101676-681$.
23 Dinerman JL, Lowenstein CJ \& Snyder SH. Molecular mechanisms of nitric oxide regulation: potential relevance to cardiovascular diseases. Circulation Research 199373 217-222.

24 Gans RO, Bilo HJ \& Donker AJ. The renal response to exogenous insulin in non-insulin-dependent diabetes mellitus in relation to blood pressure and cardiovascular hormonal status. Nephrology, Dialysis, Transplantation 199611 794-802.

Received 11 November 2000

Accepted 9 May 2001 